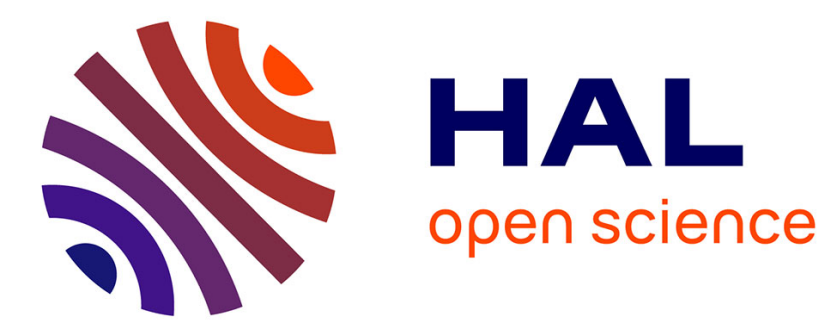

\title{
Tip Vortex Cavitation on an Oscillating Hydrofoil
}

Olivier Boulon, Jean-Pierre Franc, Jean-Marie Michel

\section{To cite this version:}

Olivier Boulon, Jean-Pierre Franc, Jean-Marie Michel. Tip Vortex Cavitation on an Oscillating Hydrofoil. Journal of Fluids Engineering, 1997, 119 (4), pp.752-758. 10.1115/1.2819494 . hal-01133765

\section{HAL Id: hal-01133765 \\ https://hal.science/hal-01133765}

Submitted on 2 Sep 2020

HAL is a multi-disciplinary open access archive for the deposit and dissemination of scientific research documents, whether they are published or not. The documents may come from teaching and research institutions in France or abroad, or from public or private research centers.
L'archive ouverte pluridisciplinaire HAL, est destinée au dépôt et à la diffusion de documents scientifiques de niveau recherche, publiés ou non, émanant des établissements d'enseignement et de recherche français ou étrangers, des laboratoires publics ou privés.

\section{(c)(1)}

Distributed under a Creative Commons Attribution| 4.0 International License 


\section{Tip Vortex Cavitation on an Oscillating Hydrofoil}

This paper discusses tests conducted in the hydrodynamic tunnel of the University of Grenoble on a 3D oscillating hydrofoil. Visualization of unsteady tip vortex cavitation indicates a strong influence of the water nuclei content. The investigation was focused on the influence of the oscillation frequency on tip vortex cavitation inception. For very low nuclei content, cavitation inception is strongly delayed as compared to the steady-state results at very small oscillation frequencies. This delay is significantly reduced by nuclei seeding. The results can be explained by assuming that the time required for the inception of cavitation in the tip vortex corresponds to the time necessary for a cavitation nucleus to be captured by the vortex core.
0. Boulon

PhD. Student.

J. P. Franc

Research Assistant.

J. M. Michel

Research Director.

Laboratoire des Ecoulements Géophysiques et Industriels, Institut de Mécanique de Grenoble, BP 53 , 38041 Grenoble Cedex 9, France

\section{Introduction}

Marine propellers generally operate under unsteady conditions. During one revolution, a propeller blade experiences varying pressure and velocity conditions because of a number of phenomena, such as the wake of the hull or the variation in submersion depth. Therefore, it is important to know the influence of unsteadiness on the inception and the development of cavitation, especially the tip vortex cavitation which generally appears first.

Tip vortex cavitation is still extensively studied (see in particular, Arndt et al., 1991, Fruman, 1995), but only a very few number of results are related to tip vortex cavitation under unsteady conditions. To our knowledge, the only results available on the subject are those of Hart et al. $(1991,1992)$ and McKenney and Hart (1993). They conducted experiments on a NACA 64A309 cross section hydrofoil of rectangular planform, oscillating sinusoidally in pitch about the center of pressure at frequencies varying between 0 and $50 \mathrm{~Hz}$, this corresponding to a reduced frequency $f *$ ranging from 0 to 2 . The mean angle of attack was 5 or 7 degrees and the oscillation amplitude \pm 2 or \pm 5 degrees. Their visualization show an intricate vortex chain resulting from the interaction between the tip vortex and the spanwise vortices shed from the trailing edge by the oscillating foil.

The present study, which is also relative to an oscillating hydrofoil, is part of a French program on tip vortex cavitation involving several laboratories. Main results in steady flow were presented by Fruman et al. (1992). Because of the large number of parameters involved in the unsteady configuration $(\sigma, \operatorname{Re}, V$, nuclei content, $f, \alpha_{0}, \Delta \alpha$ ), it is difficult to conduct a comprehensive research program. We focused our attention on a few typical questions, mainly the influence of nuclei content and oscillation frequency on the inception of tip vortex cavitation. It allowed us to analyze this type of unsteady cavitation in terms of the three characteristic times important in this problem: the oscillation period, the nucleus capture time, and the transit time $c / V$. A few additional conditions were examined briefly and are the effects of mean angle of attack and Reynolds number.

\section{Experimental Facilities}

The tests were conducted in the free surface channel of the hydrodynamic tunnel of the University of Grenoble (BriançonMarjollet and Michel, 1990). The flow velocity can vary between 3 and $12 \mathrm{~m} / \mathrm{s}$ and the turbulence intensity is about $1.5 \%$.
The hydrofoil has a symmetrical NACA 16-020 cross-section and an elliptical planform. The maximum chord length is $c=$ $60 \mathrm{~mm}$, the span length is $b / 2=90 \mathrm{~mm}$ and the aspect ratio is $A=3.8$. The dimensions of the test section and the foil arrangement are shown in Fig. 1.

The foil is oscillating sinusoidally in pitch around its midchord. The instantaneous angle of attack $\alpha(t)$ is given by $\alpha(t)$ $=\alpha_{0}+\Delta \alpha \sin 2 \pi f t$. All the results presented here correspond to an oscillation amplitude $\Delta \alpha$ equal to 5 degrees. The mean angle of attack is generally kept constant at 10 degrees, although a few tests were conducted for other values to analyse the effect of this parameter ( see Section 5). The oscillation frequency $f$ is systematically increased from 0 to $24 \mathrm{~Hz}$; the maximum value of the reduced frequency $f^{*}$, obtained for the minimum flow velocity of $3 \mathrm{~m} / \mathrm{s}$, is about 0.5 . Most tests were carried out at a Reynolds number equal to $4.5 \times 10^{5}$, i.e., at a flow velocity of about $7 \mathrm{~m} / \mathrm{s}$; the influence of Reynolds number is analyzed in Section 6.

A fundamental parameter to be controlled during the tests is the water quality which is characterized by the nuclei content. The hydrodynamic tunnel of the University of Grenoble is equipped with a nuclei seeding device which is done by the injection of water saturated with air at high pressure, and a classical cavitation susceptibility meter for nuclei measurement (Briançon-Marjollet and Michel, 1990). In the present study, two water qualities were considered: one corresponds to normal operating conditions without nuclei injection, whereas the other one corresponds to maximum nuclei injection. They are characterised by the histograms of critical pressure presented in Fig. 2 . We can roughly consider that, without nuclei seeding, there is almost no cavitation nucleus in the water, in comparison to the case with nuclei injection.

Visualization were systematically obtained for various operating conditions either by high-speed movies or by video recording under stroboscopic lighting at a frequency slightly different than the oscillation frequency.

From visualization obtained by video recording, it is possible to determine the inception of tip vortex cavitation. In the case of an oscillating foil, tip vortex cavitation appears during the oscillation cycle and, for any value of the cavitation parameter, inception is characterized by a critical value of the angle of attack.

Cavitation inception is detected visually. It is known that such a procedure may be lacking in accuracy, especially in the case of high nuclei contents for which it may be difficult to distinguish between actual cavitation and a simple migration of nuclei toward the vortex core with a limited growth. To limit the subjectivity of inception measurements, two inception criteria were defined: 


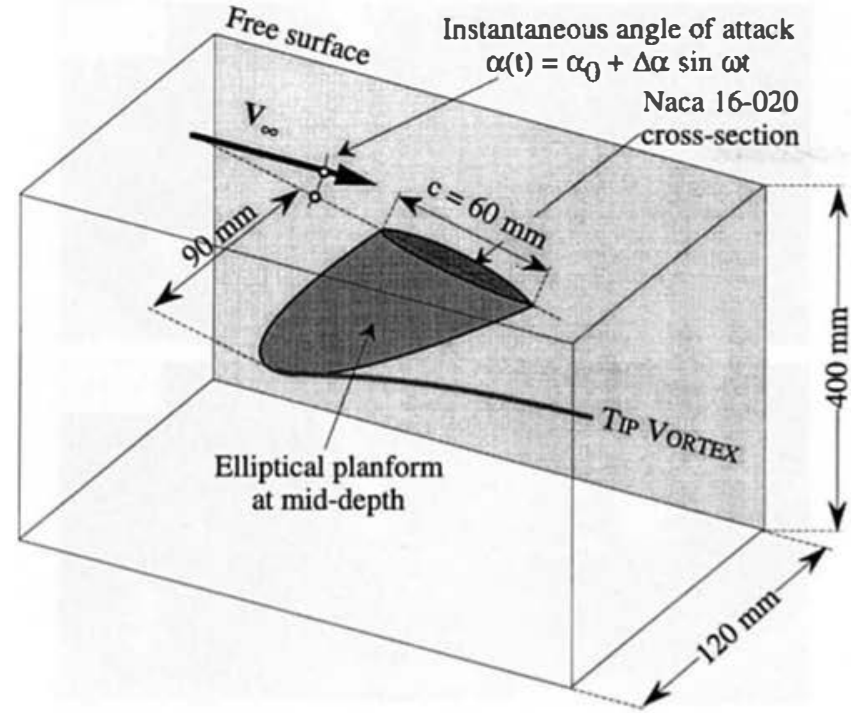

Fig. 1 Test section and foil arrangement

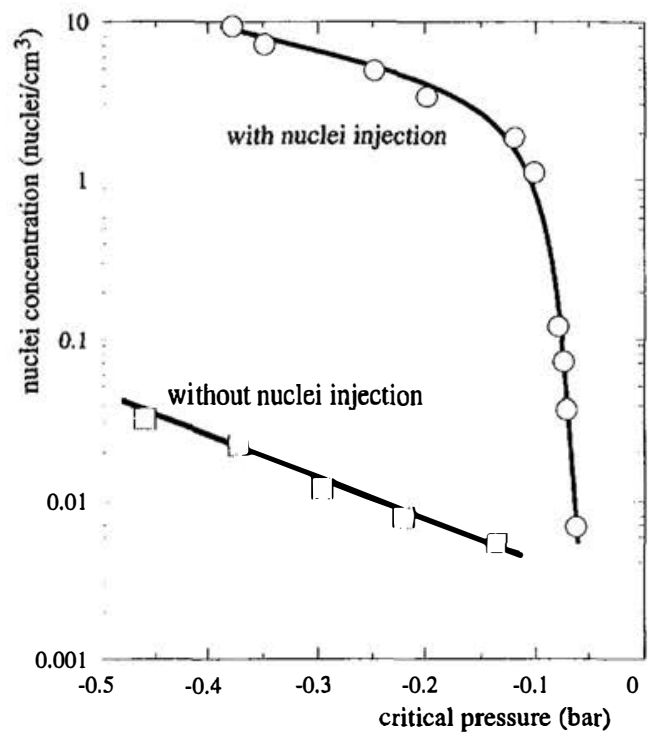

Fig. 2 Histograms of critical pressure presenting the difference in water nuclei content with and without nuclei injection for $\sigma=1.2$ and $V=7.7$ $\mathrm{m} / \mathrm{s}$

- the occurrence of intermittent cavitation along the vortex path which consists in determining the "first" cavitation bubble;
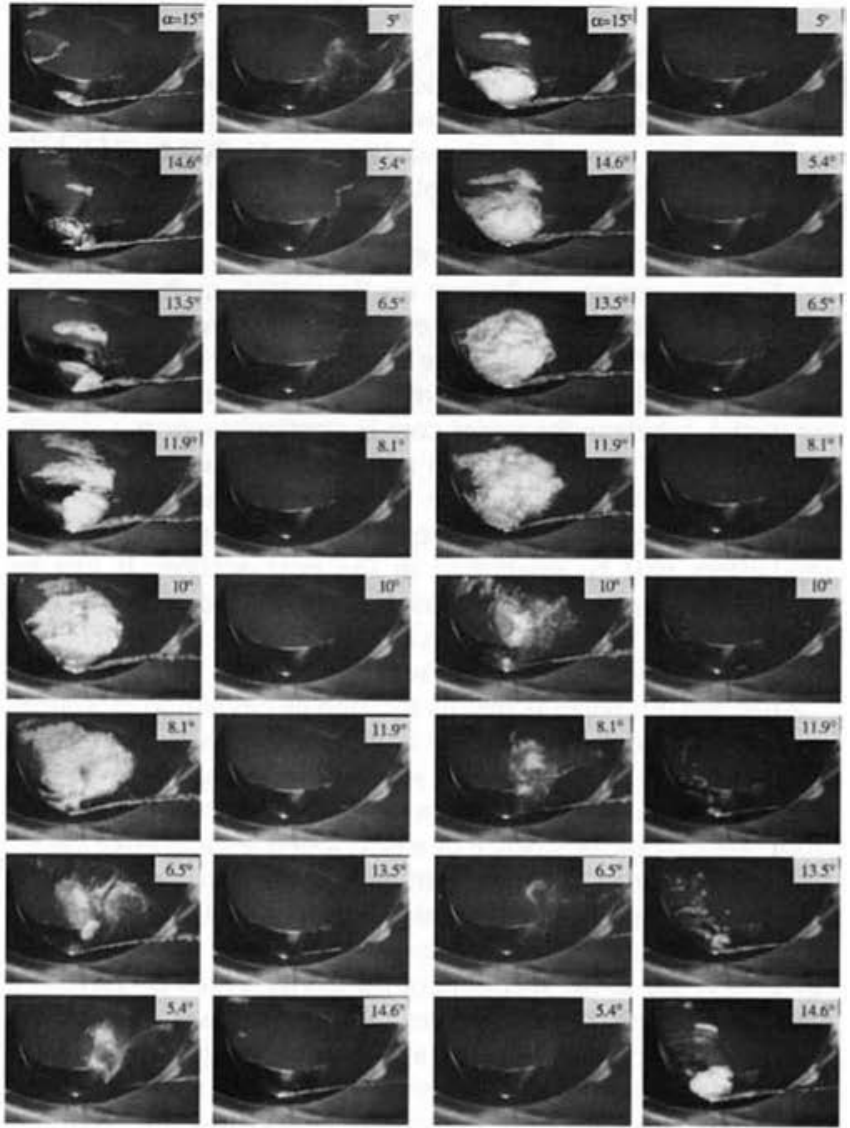

(a)
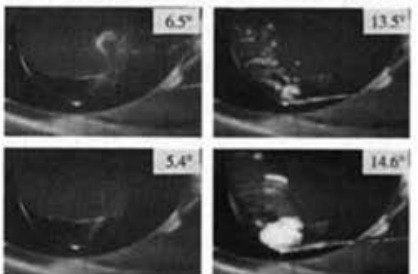

(b)

Fig. 3 Visualization of tip vortex cavitation during one period of oscillation for $\sigma=1.2, \alpha_{0}=10 \mathrm{deg}, \Delta \alpha=5 \mathrm{deg}, f=23.3 \mathrm{~Hz}$, with and without nuclei injection, at $R e=4.510^{5}$. The origin of time $t / T=0$ for the first image (upper left) of both cycles corresponds to the maximum angle of attack. The time interval between two consecutive images is $\Delta t / T=$ $\frac{1}{16}$. (a) Without nuclei injection; (b) with nuclei injection

- the occurrence of a permanent vapour core attached to the tip.

It was noticed that the second criterion was much easier to observe and much more reproducible than the first one, especially under unsteady conditions. Consequently, all the inception measurements presented are relative to this second criterion, except in Section 4.1, where a comparison of both criteria is presented under steady conditions.

\section{Visualization}

Figure 3 presents an example of visualization obtained from high-speed movies at a rate of $4000 \mathrm{frames} / \mathrm{s}$. A whole period

\section{Nomenclature}

$a=$ viscous core radius $\mathrm{m}$

$A=$ aspect ratio $A=4 b / \pi c=3.8$

$b / 2=$ span length $b / 2=90 \mathrm{~mm}$

$c=$ chord length $c=60 \mathrm{~mm}$

$f=$ oscillation frequency $\mathrm{Hz}$

$f^{*}=$ reduced oscillation frequency $f *$ $=f c / V$

$n=$ nuclei density nuclei $/ \mathrm{cm}^{3}$

$p=$ pressure $\mathrm{Pa}$

$p_{\text {ref }}=$ reference hydrostatic pressure at foil tip $\mathrm{Pa}$ $p_{v}=$ vapor pressure $\mathrm{Pa}$

$r=$ radial distance $\mathrm{m}$

$R=$ microbubble radius $m$

$\operatorname{Re}=$ Reynolds number -

$t=$ time $\mathrm{s}$

$T=$ oscillation period $T=1 / f \mathrm{~s}$

$T_{\nu}=$ viscous time $T_{\nu}=R^{2} / 4 \nu \mathrm{s}$

$V=$ flow velocity $\mathrm{m} / \mathrm{s}$

$\vec{W}=$ bubble velocity $\mathrm{m} / \mathrm{s}$

$\alpha=$ instantaneous angle of attack degree $\alpha_{0}=$ mean angle of attack degree

$\Gamma=$ vortex strength $\mathrm{m}^{2} / \mathrm{s}$

$\Delta \alpha=$ oscillation amplitude degree

$\nu=$ liquid kinematic viscosity $\mathrm{m}^{2} / \mathrm{s}$

$\rho=$ liquid density $\mathrm{kg} / \mathrm{m}^{3}$

$\sigma=$ cavitation parameter $\sigma=\left(p_{\text {ref }}-\right.$ $\left.p_{v}\right) /\left(\frac{1}{2} \rho V^{2}\right)$

$\tau=$ radial capture time $\mathrm{s}$

$\tau^{\prime}=$ axial feeding time $s$

$\omega=$ vortex rotational speed $\mathrm{rd} / \mathrm{s}$ 
of oscillation is shown in two cases: Fig. 3(a) corresponds to the case without nuclei injection, whereas Fig. $3(b)$ corresponds to the case with nuclei injection, all other parameters being unchanged. The presence of nuclei in case $3(b)$ is confirmed on images $13(\alpha=10 \mathrm{deg})$ and $14(\alpha=11.9 \mathrm{deg})$ on which we can observe cavitation bubbles exploding on the upper-side of the foil.

A careful comparison of both cases shows that the nuclei content has a strong influence on tip vortex cavitation under unsteady conditions. For instance, if we compare images 14 ( $\alpha$ $=11.9 \mathrm{deg}$ ) of both cycles, we observe only a cavitation spot approximately half a chord downstream the tip in the case without nuclei, whereas the vapor core is continuous over a significant portion of the vortex path and already attached to the tip in the case with seeding. The presence of nuclei leads to a significant advance in the inception of tip vortex cavitation. This effect will be quantified in the next section. It can be noticed that the development of the leading edge cavity also experiences a significant advance in the case of nuclei seeding, compared to the case without nuclei injection.

Although this paper is mainly focused on cavitation inception, we can observe on Fig. 3 that cavitation desinence is also in advance in the case of nuclei seeding. Whereas the influence of nuclei on inception is commonly accepted, the effect of microbubbles on the desinence of a developed leading edge cavity and on tip vortex cavitation might appear intriguing at first sight. If we observe in detail both cycles of Fig. 3, the disappearance of cavitation - tip vortex cavitation as well as leading edge cavity - is due to the shedding of the cavity which is convected downstream and then collapses. For both cases, the cavity growth process is very similar and the shedding process seems to take place as soon as the cavity has reached about the same critical size. As in the case of nuclei seeding the cavity is triggered much earlier, the whole growth and shedding processes are in advance. Thus, the desinence of cavitation is also in advance in the case of nuclei seeding.

At high reduced oscillation frequencies, the tip vortex path has a clear sinusoidal shape characterized by a wavelength $\lambda=$ $V / f$ or a reduced wavelength $\lambda / c=1 / f^{*}$ as shown on Fig. 4 . It is made of a sequence of noncavitating and cavitating pieces according to the phase of their emission in the oscillation cycle. If we look at the vortex tip in more details, we can observe that the cavitating vortex becomes thicker near the tip and changes into a twisted ribbon (Fig. 5, $\alpha=14 \mathrm{deg}$ ). Then it seems to split (Fig. 5, $\alpha=15 \mathrm{deg}$ ) and to give birth to a secondary cavitating vortex (Fig. 5, $\alpha=12,10$ and $6 \mathrm{deg}$ ) of rather spanwise nature, contrary to the tip vortex which is fundamentally a streamwise structure.

The formation of this secondary vortex is difficult to interpret. According to Arndt et al. (1991), who reported similar observations in the steady case, it may originate in a difference of vorticity associated to a separation bubble of varying length. It may also be connected to the classical mechanism of shedding

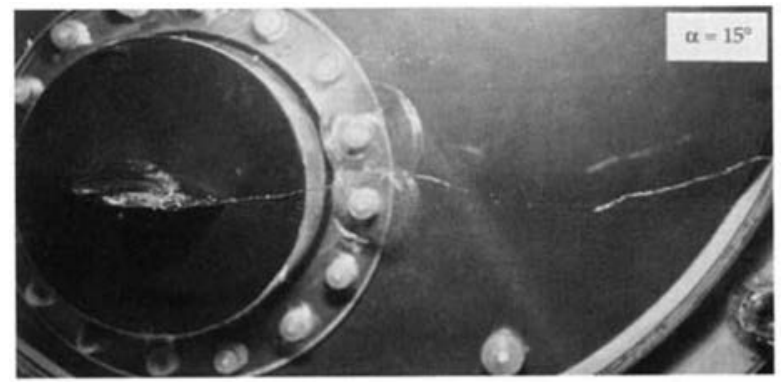

Fig. 4 Overall view of the sinusoidal tip vortex path downstream the oscillating hydrofoil at high reduced oscillation frequency with nuclei injection. $\sigma=1, \mathrm{Re}=210^{5}, f=23.3 \mathrm{~Hz}, f^{\star}=0.4, \alpha_{0}=10 \mathrm{deg}, \Delta \alpha=$ 5 deg
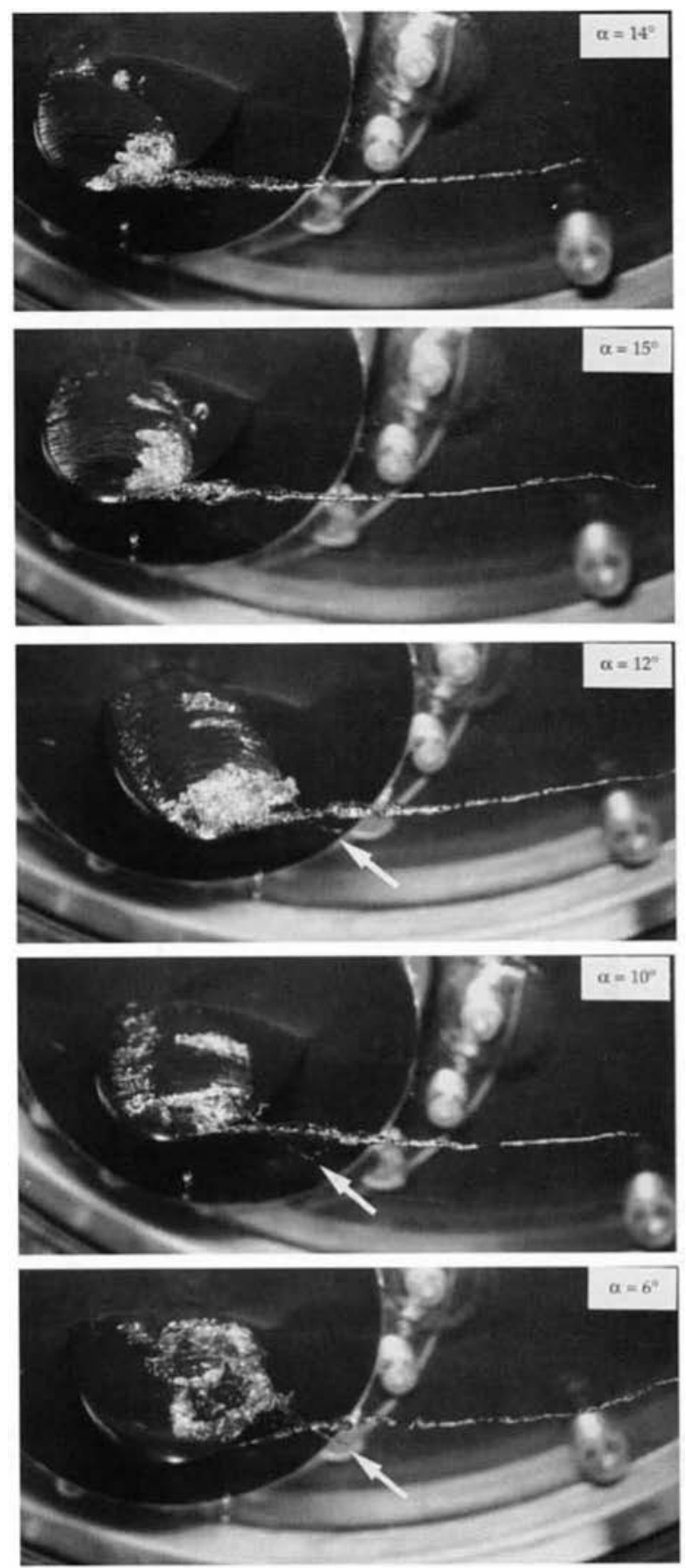

Fig. 5 Visualization of tip vortex cavitation at high reduced oscillation frequency, with nuclei injection, $\sigma=1, R e=210^{5}, f=23.3 \mathrm{~Hz}, f^{\star}=$ $0.4, \alpha_{0}=10 \mathrm{deg}, \Delta \alpha=5 \mathrm{deg}$

of spanwise vortices by a partial cavity. Anyhow, the greatest care must be taken in the interpretation of visualization by cavitation in so far as a non cavitating - and so invisiblevortex is very likely to pre-exist to the vapor structure.

\section{Tip Vortex Cavitation Inception}

4.1 Steady Case. Figure 6 shows that, in steady flow, the incipient cavitation number defined on the basis of the vapor core getting attached to the tip does not depend upon the nuclei content. On the contrary, in the case of nuclei injection, the "first" bubble appears before the case without nuclei injection.

Results presented in Fig. 6 are independent of the experimental procedure i.e., operating either at fixed $\sigma$ and increasing $\alpha$ 


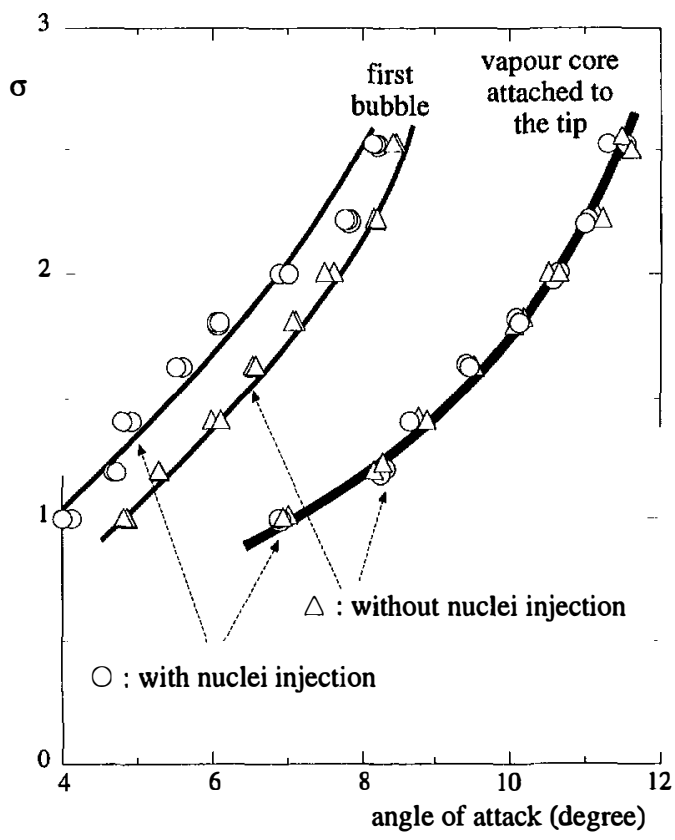

Fig. 6 Incipient cavitation numbers versus the angle of attack for $\mathrm{Re}=$ 4.5 $10^{5}$ with and without nuclei injection

or at fixed $\alpha$ and decreasing $\sigma$. In addition, no hysteresis is observed between inception and desinence.

4.2 Unsteady Case. Figure 7 presents the angle of attack for which the vapor core gets attached to the tip (called incipient angle of attack $\alpha_{i}$ ) versus the reduced frequency for $\sigma=1.6$ and for the two water qualities considered in the present study. The case $f^{*}=0$ corresponds to the steady state for which we already noticed that no nuclei effect exists if the second inception criterion is used. For this reason both curves start from the same point.

In the case of nuclei seeding, when the reduced oscillation frequency increases, the incipient angle of attack gradually increases. For the higher reduced frequency $f^{*}=0.2$ considered in Fig. 7, the inception of tip vortex cavitation is delayed about 3 degrees in comparison with the quasi-steady case and is about 2 degrees in advance with respect to the case without nuclei seeding at the same frequency.

Without nuclei injection, we observe on Fig. 7 a strong unsteady effect for very small values of the reduced frequency $f^{*}$ $<0.01$, characterized by an inception delay of more than 4 degrees. On the contrary, for values of the reduced frequency greater than about 0.05 , no unsteady effect is visible on tip

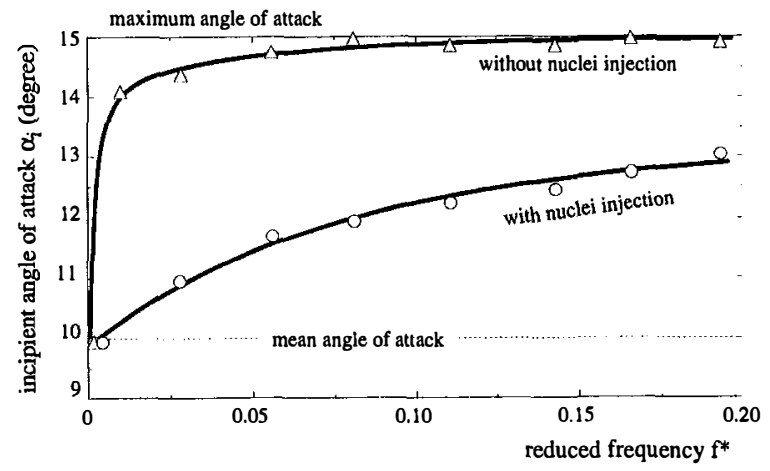

Fig. 7 Incipient angle of attack as a function of reduced frequency for $\sigma=1.6, \alpha_{0}=10$ degrees, $\Delta \alpha=5$ degrees, with and without nuclei injection, at $R e=4.510^{6}$

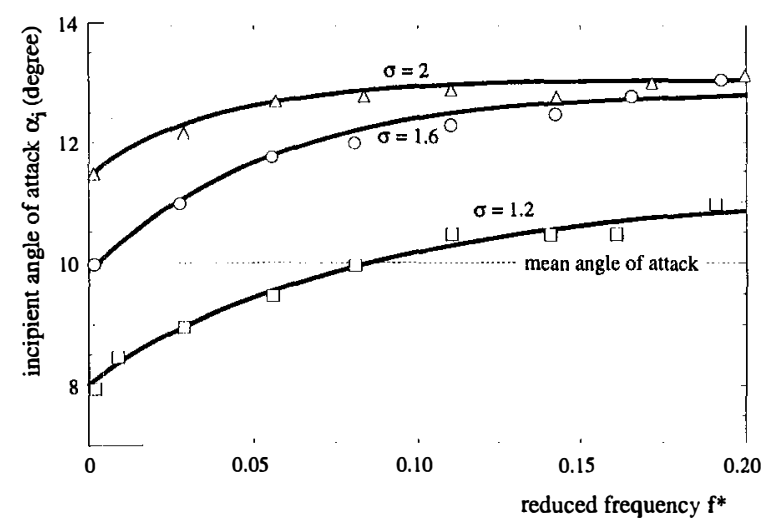

Fig. 8 Incipient angle of attack as a function of reduced frequency for $\sigma=2,1.6$, and 1.2, $\alpha_{0}=10$ degrees, $\Delta \alpha=5$ degrees, with nuclei injection at $\mathrm{Re}=4.510^{5}$-Influence of cavitation number.

vortex cavitation; it always occurs around the maximum angle of attack.

For a higher value of the cavitation parameter equal to 2 , the incipient angle of attack also increases gradually with the oscillation frequency in the case of nuclei seeding. The difference is that the tip vortex does not cavitate without nuclei injection. A synthesis of the values of the incipient angle of attack versus the reduced frequency for various values of the cavitation number is given on Fig. 8. Lower values of the cavitation number $(\sigma<1)$ were not investigated in detail because cavitation is much more developed and a leading edge cavity strongly interacts with the tip vortex.

We emphasize on the observation that, as soon as the foil oscillates, i.e., for an oscillation frequency as small as $1 \mathrm{~Hz}$, an important delay of about 4 degrees is observed without nuclei injection. This delay is considerably reduced if the flow contains a high enough nuclei concentration. Therefore, the effect of the reduced frequency on the incipient angle of attack, which is shown in Fig. 7, appears to be a combined effect of unsteadiness and water quality. This question will be discussed in more details in Section 7.

\section{Effect of Mean Angle of Attack}

Figure 9 illustrates the influence of the mean value of the angle of attack $\alpha_{0}$ on tip vortex cavitation inception. Two different values of $\alpha_{0}$ were chosen and the oscillation amplitude was kept constant at 5 degrees.

For a given water quality, cavitation inception appears to be delayed by an increase of the mean angle of attack. It is satisfactory to find out that this delay vanishes as the reduced oscillation frequency approaches zero, i.e. under quasi-steady conditions.

\section{Effect of Reynolds Number}

The effect of Reynolds number was investigated by varying the flow velocity. Table 1 gives a few results in a particular case. It clearly appears that the Reynolds number has no significant influence on the inception of tip vortex cavitation under unsteady conditions, at least in its limited range of variation presently investigated.

\section{Discussion of Results}

Let us consider again the results related to the case without nuclei injection presented in Section 4.2 (in particular Fig. 7). The question is to interpret why there is such an important influence of the oscillation for reduced frequencies as small as 0.01 . 


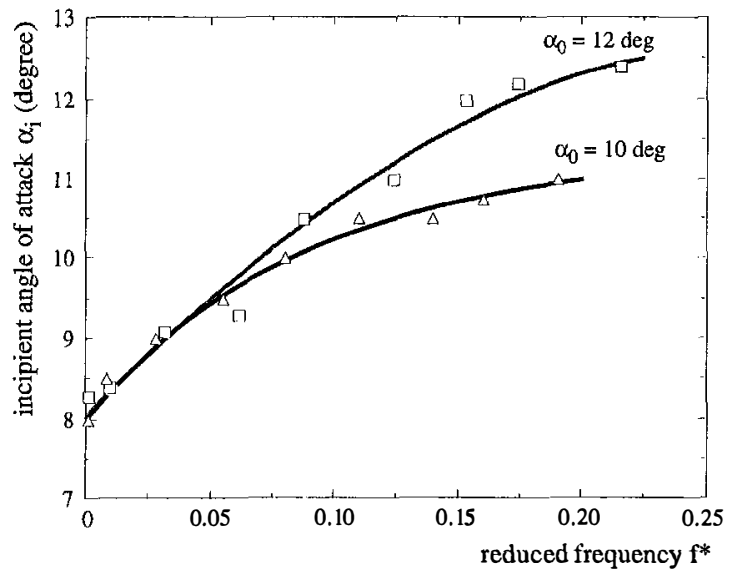

Fig. 9 Incipient angle of attack as a function of reduced frequency for $\sigma=1.2, \Delta \alpha=5$ degrees, with nuclei injection, at $R e=4.510^{5}-$ Influence of mean angle of attack.

A priori, we could suspect a purely unsteady effect affecting, in particular, the setting of the vortex strength. The reduced frequency, which is defined by:

$$
f^{*}=\frac{f c}{V}=\frac{c / V}{T}
$$

measures the ratio of the time required for a fluid particle to go over the chord length, to the period of oscillation. If $f^{*}=0.01$, the displacement of the foil can be neglected during the transit time $T / 100$ of a particle along the chord length: the fluid particle will indeed not feel the foil movement during its travel. We can then expect that quasi-steady conditions are achieved, and tip vortex cavitation should appear for a value of the angle of attack close to the one corresponding to the steady case. Then, unsteadiness cannot be the major reason for such an inception delay at very low reduced frequencies.

Furthermore, in steady flow and without nuclei seeding, if the foil is rapidly moved from 0 degree to the angle of attack corresponding to tip vortex cavitation inception (for a given $\sigma$ value), we observe the inception of cavitation after a delay, which is variable and of the order of a few seconds, and in any case much larger than $c / V$. Then, it is clear that tip vortex cavitation requires some time to appear after the flow is settled.

Considering present experimental results on the influence of water quality in unsteady flow, we are led to conjecture that this inception time is the time required for a nucleus to enter the vortex core. According to this interpretation, the effect of the reduced frequency presented in Section 4.2 appears to be a combined effect of water quality and unsteadiness.

Two different mechanisms are possible, for a nucleus to reach the vortex core. A nucleus can be radially captured by the tip vortex because of its rotational speed, or, it can feed the vortex core from upstream in an axial way. Both assumptions are examined hereafter.

7.1 Radial Capture. A microbubble of constant radius $R$ moves toward the vortex core with a velocity $\vec{W}$ according to the classical Johnson and Hsieh equation (Ligneul and Latorre, 1989):

$$
\frac{d \vec{W}}{d t}=-3 \frac{\operatorname{grảd} p}{\rho}-9 \frac{\nu}{R^{2}}(\vec{W}-\vec{V}) .
$$

This equation of motion of the microbubble takes into account the added mass of the bubble, the pressure forces exerted by the rotating flow and the drag evaluated on the basis of the Stokes formula. $\vec{V}$ is the purely tangential flow velocity induced by the tip vortex and $\mid$ grảd $p \mid=\rho V^{2} / r$ the corresponding pressure gradient at a radial distance $r$ from the core.

We suppose that the nucleus radius $R$ is small enough, so that the term on the left-hand side can be neglected in comparison with the second term on the right-hand side. It is equivalent to suppose that the viscous time $T_{\nu}$ defined by $T_{\nu}=R^{2} / 4 \nu$ is much smaller than the nucleus capture time denoted by $\tau$. This assumption, which will be checked a posteriori, leads to the following simplified equation:

$$
\vec{W}-\vec{V}=-\frac{R^{2}}{3 \nu} \frac{\operatorname{grảd} p}{\rho} .
$$

Equation (2) simply means that the pressure forces exactly balance the viscous drag at any time. As a result, the microbubble has a tangential velocity component equal to the flow tangential velocity, and a centripetal velocity component given by:

$$
-\frac{d r}{d t}=\frac{R^{2}}{3 \nu} \frac{V^{2}}{r} .
$$

The tangential flow velocity in the tip vortex is given by:

$$
V=\frac{\Gamma}{2 \pi r}
$$

where $\Gamma$ denotes the vortex strength. By integrating Eq. (3) we get the instantaneous radial position $r$ of the micro bubble as a function of time $t$ and of its initial position $r_{0}$ :

$$
r^{4}-r^{4}=\frac{4}{3} \frac{R^{2}}{\nu} \frac{\Gamma^{2}}{4 \pi^{2}} t
$$

The capture time $\tau$ is the time necessary for the bubble to reach the vortex core $r=0$. We get from Eq. (5):

$$
\tau=\frac{3}{4} \frac{\nu}{R^{2}} \frac{4 \pi^{2}}{\Gamma^{2}} r_{0}^{4}
$$

If we consider a classical Rankine vortex of core radius, $a$, and rotational speed, $\omega$, the vortex strength is given by $\Gamma$ $2 \pi \omega a^{2}$ and finally, the capture time is:

$$
\tau=\frac{3 \nu r_{0}^{4}}{4 \omega^{2} R^{2} a^{4}}
$$

The parameters $a$ and $\omega$ can be estimated from LDV measurements. Typical values in the present case (Fruman et al., 1992) are $a=0.9 \mathrm{~mm}$ and $\omega=10000 \mathrm{rd} / \mathrm{s}$. These values are characteristic of the steady case and are used in the present unsteady configuration to get estimates of capture times.

The distance $r_{0}$ between the vortex centre and the nearest nucleus highly depends upon the nuclei density. Cavitation inception generally occurs on a limited part of the vortex, in the vicinity of the tip. For a rough estimate, we shall suppose that inception can occur on a length of the order of the chord length $c$. If the nuclei density is $n$, a cylinder of length $c$ and radius $r_{\max }$ defined by:

Table 1 Influence of Reynolds number on tip vortex cavitation inception for $\sigma=1.6, \alpha_{0}=10$ degrees, $\Delta \alpha=5$ degrees, $f^{*}=0.1$, with nuclei injection

\begin{tabular}{lrrr}
\multicolumn{1}{c}{$\operatorname{Re}$} & $1.810^{5}$ & $4.610^{5}$ & $7.410^{5}$ \\
$V(\mathrm{~m} / \mathrm{s})$ & 2.7 & 6.7 & 10.8 \\
$f(\mathrm{~Hz})$ & 4.5 & 11.1 & 18.1 \\
$\alpha$ at inception $\left(^{\circ}\right)$ & 11.1 & 11.4 & 11.2
\end{tabular}


Table 2 Estimates of the capture time for two different water qualities

$\begin{array}{lcc} & \begin{array}{c}\text { Without nuclei } \\ \text { injection }\end{array} & \begin{array}{c}\text { With nuclei } \\ \text { injection }\end{array} \\ n\left(\text { nuclei } / \mathrm{cm}^{3}\right) & 0.05 & 10 \\ r_{0}(\mathrm{~mm}) & 6.8 & 0.49 \\ \tau & 0.98 \mathrm{~s} & 26 \mu \mathrm{s} \\ & \\ & r_{\max }=\frac{1}{\sqrt{\pi n c}}\end{array}$

contains, on an average, one nucleus. Then, the mean value of the distance of a nuclei to the vortex core is $r_{0} \approx \frac{2}{3} r_{\max }$.

From Fig. 2, the nuclei concentrations can be chosen equal to about 0.05 and 10 for the two water qualities. If we consider a typical nucleus radius $R=5 \mu \mathrm{m}$, we get the rough estimates presented in Table 2.

As the nuclei moves toward the vortex core, it goes through decreasing pressures and grows. The present computation does not take into account this phenomenon as the nucleus radius is supposed constant in Eq. (1).

Let us notice that the viscous time given as $T_{\nu}=R^{2} / 4 \nu$ is equal to $6 \mu$ s and is smaller than the capture time $\tau$, as assumed a priori. The assumption is less proper in the case of nuclei seeding.

7.2 Axial Feeding. In the case of an axial feeding, the nucleus enters the vortex core from upstream through a circular section of radius $a$. If the nuclei density is $n$, a cylinder of radius $a$ and length $l$ defined by:

$$
l=\frac{1}{n \pi a^{2}}
$$

contains, on an average, one nucleus. Then, the mean time $\tau^{\prime}$ for a nucleus to axially feed the vortex core is of the order of:

$$
\tau^{\prime}=\frac{l}{V}=\frac{1}{n \pi a^{2} V}
$$

Considering again the orders of magnitude of nuclei concentrations given in Table 2, we get, for $a=0.9 \mathrm{~mm}$ and $V=7.7$ $\mathrm{m} / \mathrm{s}$ :

- $\tau^{\prime} \approx 1 \mathrm{~s}$ without nuclei injection

- $\tau^{\prime} \approx 5 \mathrm{~ms}$ with nuclei injection.

7.3 Interpretation of Experimental Results. Whatever may be the feeding mechanism (radial or axial), the orders of magnitude presented in Sections 7.1 and 7.2 show that, without nuclei injection, the time required for a microbubble to reach the vortex core and initiate cavitation is of the order of one second whereas it is much smaller in the case of nuclei injection, due to the large increase in nuclei concentration.

If the period of oscillation is small with respect to this capture time, there will not be enough time for a nucleus to reach the vortex core and trigger cavitation during each cycle of oscillation. In that case, a significant deviation from the quasi-steady case is expected. On the contrary, if the period of oscillation is much larger than the capture time, cavitation is quasi instantaneously triggered.

Even though some very crude assumptions were made, the above-mentioned values give a satisfactory account of the experimental results presented in Section 4.2. It can be noticed that, in the case of nuclei seeding, the capture times $\tau$ and $\tau^{\prime}$ are so small that no nuclei effect is expected in practice; nevertheless, a purely unsteady effect (i.e., not connected with the water quality but affecting for instance the instantaneous vortex strength) is expected for reduced oscillation frequencies getting near one. It is the reason for the progressive increase in cavitation inception delay observed for $f^{*}<0.2$, which is quite different in nature for the one observed for $f^{*} \cong 0.01$ without nuclei injection.

\section{Conclusions}

The inception of tip vortex cavitation on an oscillating hydrofoil strongly depends upon the oscillation frequency and the nuclei concentration.

- For very low nuclei content, an important delay (in comparison with the steady flow) is observed for reduced oscillation frequencies as small as 0.01 . At higher frequencies, this delay tends to become independent of the oscillation frequency, cavitation inception occurring close to the maximum angle of attack.

- The injection of nuclei significantly decreases the delay observed for $f^{*} \cong 0.01$ without nuclei seeding. In addition, the delay increases much more gradually as the reduced oscillation frequency increases.

The unsteady effect discussed in the present study is closely correlated to the nuclei concentration. More precisely, cavitation will occur in the core of the tip vortex only if a nucleus has enough time to reach the core during the period of oscillation and trigger cavitation.

- If the nuclei concentration is so small that the capture time is much higher than the period of oscillation, a combined effect of water quality and unsteadiness is expected. It generates a significant delay at reduced oscillation frequencies, much smaller than one.

- On the contrary, if the nuclei concentration is so high that the capture time is negligible in comparison with the period of oscillation, we have to do with a purely unsteady effect which becomes significant when the period of oscillation approaches the transit time $c / V\left(f^{*} \approx 1\right)$.

In conclusion, from a fundamental viewpoint, tip vortex cavitation inception on an oscillating hydrofoil is essentially controlled by three characteristic times: the oscillation peried, the nucleus capture time, and the transit time $c / V$. In particular, the delay in the inception of cavitation strongly depends upon the relative orders of magnitude of these three characteristic times. From an experimental viewpoint, the present study confirms the fundamental importance of controlling the water quality, especially for the investigation of unsteady cavitating flows.

\section{Acknowledgments}

This work was conducted with the financial support of the "Direction des Recherches, Etudes et Techniques" (DRET) and is part of a program "Action Concertée Cavitation", headed by DRET (contract No 92-080).

\section{Experimental Uncertainties}

The uncertainties on incipient cavitation numbers and incipient angles of attack are respectively of the order of \pm 0.05 and $\pm 0.2 \mathrm{deg}$. These estimates result from various series of tests carried out independently by several experimentators. The uncertainties on the determination of flow parameters (as flow velocity and pressure) are negligible in comparison with differences of visual appreciation of cavitation inception or defaults of reproducibility.

\section{References}

Arndt. R. E. A. and Keller A. P., 1991, "Water Quality Effect on Cavitation Inception on a Trailing Vortex," ASME-JSME Fluids Engineering Conference, Portland, Oregon, June 23-27, FED-Vol. 116, pp. I-9. 
Arndt R. E. A., Arakeri V. H. and Higuchi H., 1991, "Some Observations of Tip-Vortex Cavitation," Journal of Fluid Mechanics, Vol. 229, pp. 269289

Briançon-Marjollet L., and Michel J. M., 1990, "The Hydrodynamic Tunnel of I.M.G.: Former and Recent Equipments," ASME JourNaL OF FLuIDS ENGINEERING, Vol. 112, pp. 338-342.

Fruman D. H., 1995, "The «Action Concertée Cavitation》 Program and Accomplishments," International Symposium on Cavitation, 2-5 May, Deauville. France.

Fruman D. H., Dugué C., Pauchet A., Cerrutti P., and Briançon-Marjollet L., 1992, "Tip Vortex Roll-Up and Cavitation," 19th Symposium on Naval Hydrodynamics, Seoul, Korea, Aug.
Hart D. P., 1991, "Cavitation Inception in the Tip Vortex Region of an Oscillating Hydrofoil," 1991 ASME Cavitation and Multiphase Flow Forum, FED-Vol. 109, pp. 35-41.

Hart D. P., Acosta A., and Leonard A., 1992, "Observation of Cavitation and Wake Structure of Unsteady Tip Vortex Flows," International STG Symposium on Propulsors and Cavitation, June 22-24, Hamburg, Germany.

Ligneul P., and Latorre R., 1989, "Study on the Capture and Noise of Spherical Nuclei in the Presence of the Tip Vortex of Hydrofoils and Propellers," Acoustica, Vol. 68, pp. 1-14.

McKenney E. A. and Hart D. P., 1993, "Experimental Determination of Bound Circulation and Shed Vorticity Induced by an Oscillating Hydrofoil,' 1993 ASME Cavitation and Multiphase Flow Forum, FED-Vol. 153, pp. 87-91. 